\title{
Lingual abscess in a heavy smoker
}

\author{
Ilker Akbas, Abdullah Osman Kocak*, Fatma Cakmak and Burak Katipoglu \\ Department of Medical Faculty, Ataturk University, Erzurum, Turkey
}

\begin{abstract}
Lingual abscess is an extremely rare disease that misdiagnose may lead to sepsis and/or airway obstruction. A 50-year-old male patient was admitted to our emergency service with complaints of swelling of his tongue, pain and dysphagia. The swelling and pain had started 4 days ago and had been increasing gradually. Additionally, over the last 24 hours, dysphagia and difficulty in speaking developed. He was a heavy smoker (2 packets/day for 35 years). His medical history revealed neither a predisposing factor such as a chronic disorder including immunosuppression nor recent history of oral trauma. His physical examination revealed poor oral hygiene and a red, swollen tongue. There were two painful, protuberant lesions with purulent discharge: one located over the tongue and the other located sublingually. He had hoarse voice and saliva was discharging from his mouth. He had fever, leukocytosis and elevated C-reactive protein. Fine needle aspiration was performed and broad spectrum antibiotics were given. The culture result revealed gram-positive cocci and anaerobes. The patient was discharged at his $8^{\text {th }}$ day of admission, following regression of his symptoms. In conclusion, this case leads us to suggest that smoking may be an etiological factor in the development of lingual abscess in patients with no other identifiable etiological factors. Although clinical explanations related to lingual abscess are not generally mentioned in the majority of classical essential textbooks, emergency service physicians and head-neck surgeons should be aware of this situation which may lead to fatal consequences.
\end{abstract}

\section{Introduction}

Lingual abscess is a potentially life-threatening clinical situation [1]. Only 24 cases were reported during the last century in the United States of America. It is a serious situation as delayed or missed diagnosis due to its rarity may lead to sepsis and/or airway obstruction [2]. Here, we present a case with lingual abscess with no identifiable etiological cause. We suggest that the reason may be mucosal damage related to smoking.

\section{Case}

A 50-year-old male patient was admitted to our emergency service with complaints of swelling of his tongue, pain and dysphagia. The swelling and pain had started 4 days ago and had been increasing gradually. Additionally, over the last 24 hours, dysphagia and difficulty in speaking developed. He was a heavy smoker (2 packets/day for 35 years). His medical history revealed neither a predisposing factor such as a chronic disorder including immunosuppression nor recent history of oral trauma. His physical examination revealed poor oral hygiene and a red, swollen tongue. There were two painful, protuberant lesions with purulent discharge: one located over the tongue, $2 \times 3 \mathrm{~cm}$ in dimension and the other located sublingually, $1 \times 2 \mathrm{~cm}$ in dimension (Figure 1). He had hoarse voice and saliva was discharging from his mouth. Physical examination findings of other systems were normal. His vital signs were in normal range except fever $\left(38.1^{\circ} \mathrm{C}\right)$. His laboratory results were normal except leukocytosis (WBC: $11000 / \mathrm{mm}^{3}$ reference range: 4300-10300) and elevated C-reactive protein level (120 $\mathrm{mg} / \mathrm{L}$, reference range: $0-5$ ). Maxillofacial and cervical tomographic examinations with contrast (Iopromid, Ultravist-300 vial ${ }^{\circ}$ ) were performed for differential diagnosis of retropharyngeal abscess, lingual abscess and dysphagia. These tomographic examinations revealed a lesion with peripheral contrast uptake, located at the mid-portion of the tongue and consistent with an abscess (Figure 2). The patient was consulted with an otorhinolaryngologist and then hospitalized. Here, fine needle aspiration was performed for diagnostic and therapeutic purposes and pus was drained from the lesion; then, ceftriaxone $2 \mathrm{gr} /$ day IV and ornidazole $1 \mathrm{gr} /$ day were started empirically.
The culture result revealed gram-positive cocci and anaerobes. The empirical antibiotic treatment was continued with the same protocol. The patient was discharged from the hospital at his $8^{\text {th }}$ day of admission, following regression of his symptoms.

\section{Discussion and conclusion}

This is the first case report of a patient with lingual abscess whose etiologic factor is poor oral hygiene possibly due to heavy smoking. Thus, we speculate that heavy smoking should be included as an etiologic factor.

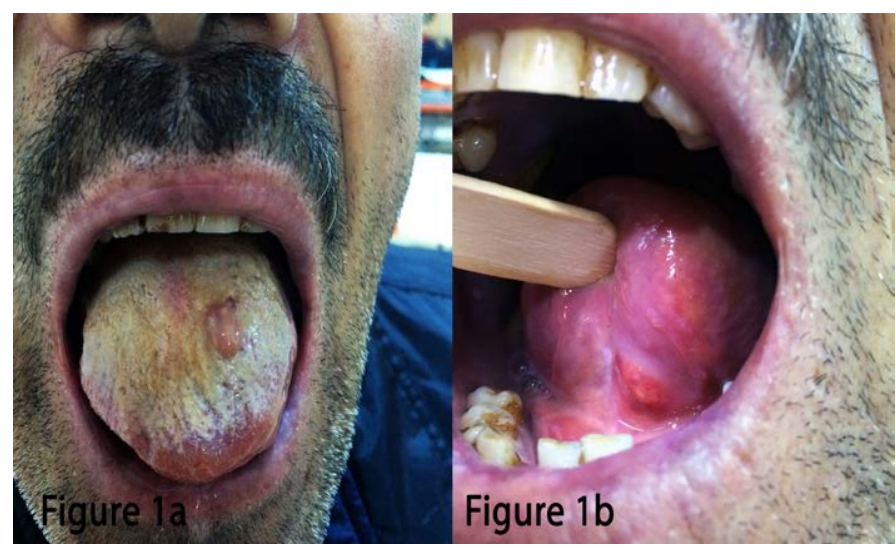

Figure 1. Dimension over the tongue.

Correspondence to: Abdullah Osman Kocak, Department of Emergency Medicine, Ataturk University, Erzurum-25240, Turkey, Tel: +90 442 3448390; Fax: +90 442 3443133; E-mail: abdullahmrym86@gmail.com

Key words: lingual abscess, smoking, odynophagia

Received: June 01, 2017; Accepted: June 24, 2017; Published: June 26, 2017 


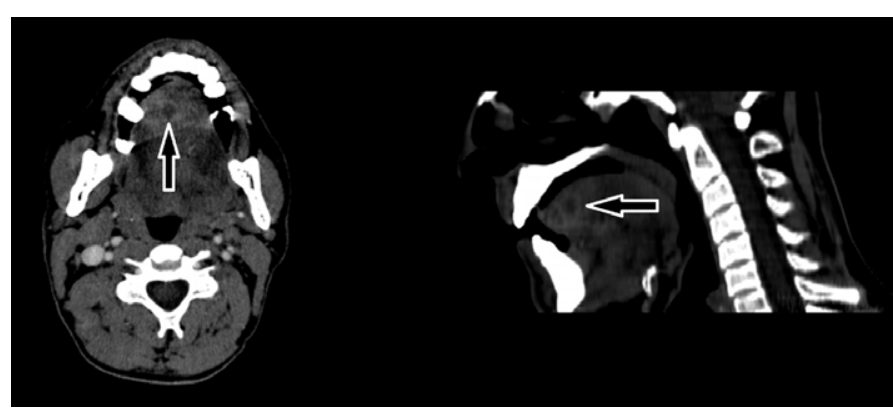

Figure 2. Contrast enhanced CT of tongue.

Although the tongue is in contact with numerous pathogens, it is highly resistant to infections. Thick keratinized mucosal layer, which is resistant to penetration of microorganisms, bulky muscle tissue, rich vascular and lymphatic drainage, cleaning effect of constant motion of the tongue and immunological properties of saliva lead to the high resistance of tongue against infections [3]. The infection rate increases particularly when integrity of tongue mucosa, which functions as mechanical barrier against microorganisms and first line of defense, is impaired [1].

Smoking increases the sensitivity to oral mucosal infections [4]. It reduces the immunological response of the host by altering the immune system in the oral, nasal and airway mucosa [5]. The development of infection is more evident in cases who have poor oral hygiene or diabetes mellitus and immunocompromised status seen in disorders such as leukaemia, neutropenia and AIDS, accompanying heavy smoking [6]. We consider that smoking caused impairment in the mucosa leading to be infected by elements of oral flora in our case.

Lingual abscess are classified according to its locations. Anterior lingual abscesses are seen more frequently, have milder clinical courses and are more easily diagnosed. Due to the risk of airway obstruction, posterior lingual abscesses are more important $[7,8]$.

Immunocompromised status, poor oral hygiene, chemotherapeutic agents and diabetes mellitus are among predisposing factors for development of lingual abscess [3]. Irregular or rotten teeth, improper denture prostheses, trauma caused by bites or foreign bodies such as fish bones, and body piercings are the most common etiological factors for the anterior abscesses [7]. It is essential to evaluate underlying medical problems and trauma. However, no specific cause can be identified in some cases [3]. No history of trauma, no history of a chronic disorder, foreign body or bite was present in our case with anterior abscess.

The clinical features of lingual abscess are fever, pain and swelling of the tongue, odynophagia, difficulty in speaking and swallowing together with a change of voice [2]. When the disease worsens, it may lead to dyspnea and dysphagia, necessitating urgent decompression [3].
Maintenance of the airway patency based on the location of the abscess, administration of antibiotics and drainage of the abscess are essential for the treatment of lingual abscesses [9]. Since staphylococci, streptococci, haemophilus, bacteroides and anaerobic species which are present within the oral flora are responsible for abscess development, empirical antibiotic treatment should be effective against these microorganisms. However, mixt infections should also be taken into consideration $[2,9]$.

While medical treatment may be sufficient for abscesses located at the anterior portion of the tongue, surgical intervention should absolutely be performed in abscesses localized at the posterior portion in order to prevent infection from spreading to deep neck tissues [8]. In our case, abscess was partially drained by aspiration for the purpose of microbial culture.

In conclusion, this case leads us to suggest that smoking may be an etiological factor in the development of lingual abscess in patients with no other identifiable etiological factors. Although clinical explanations related to lingual abscess are not generally mentioned in the majority of classical essential textbooks, emergency service physicians and headneck surgeons should be aware of this situation which may lead to fatal consequences.

\section{References}

1. Antoniades K, Hadjipetrou L, Antoniades V, Antoniades D (2004) Acute tongue abscess. Report of three cases. Oral Surg Oral Med Oral Pathol Oral Radiol Endod 97: 570-573. [Crossref]

2. Kettaneh N, Williamson K (2014) Spontaneous lingual abscess in an immunocompromised patient. Am J Emerg Med 32: 492 e1-e2. [Crossref]

3. Ozgur GT, Akdogan MV, Unler GK, Gokturk HS (2015) A rare cause of acute Dysphagia: abscess of the base of the tongue. Case Rep Gastrointest Med 2015: 431738. [Crossref]

4. Qian YJ, Wang X, Gao YF, Duan N, Huang XF, Sun FF, et al. (2015) Cigarette smoke modulates nod1 signal pathway and human $\beta$ defensins expression in human oral mucosa. Cell Physiol Biochem 36: 457-473. [Crossref]

5. Lee J, Taneja V, Vassallo R (2012) Cigarette smoking and inflammation: cellular and molecular mechanisms. J Dent Res 91: 142-149. [Crossref]

6. Sanchez Barrueco A, Melchor Diaz MA, Jimenez Huerta I, Millan Juncos JM, Almodovar Alvarez C (2012) Recurrent lingual abscess. Acta Otorrinolaringol Esp 63: 318-320.

7. Kulkarni CD, Verma AK, Kanaujia R (2013) A rare case of hemilingual abscess in a 17-year-old girl: the ease of ultrasound and the advantage of MRI. Jpn J Radiol 31: 491-495. [Crossref]

8. Narasimha A, Rekha PR, Prasad CSBR (2014) Tongue (Lingual) Abscess: Two Case Reports with Review of Literature. Oral Maxillofac Pathol J 5: 456-458.

9. Vellin JF, Crestani S, Saroul N, Bivahagumye L, Gabrillargues J, Gilain L (2011) Acute abscess of the base of the tongue: a rare but important emergency. J Emerg Med 41: e107-e110. [Crossref]

Copyright: (C2017 Akbas I. This is an open-access article distributed under the terms of the Creative Commons Attribution License, which permits unrestricted use, distribution, and reproduction in any medium, provided the original author and source are credited. 